\title{
The attract force equation of energy
}

\author{
Yan Ji \\ School of Chemistry and Chemical Engineering, Southeast University, Nanjing 211189, China
}

Email address:

jiyan98@163.com

\section{To cite this article:}

Yan Ji. The Attract Force Equation of Energy. American Journal of Modern Physics. Vol. 3, No. 6, 2014, pp. 224-226. doi: 10.11648/j.ajmp.20140306.13

\begin{abstract}
The attract force equation of energy deduced from the Newton the principle of gravitation and the mass-energy equation $\left(E=\mathrm{mc}^{2}\right)$ of Einstein. The equation describe the attract force cause by two energy fields. It can be said that the energy exists in any form (such as energy of heat, energy of photon, electronic energy, magnetic energy, even kinetic energy, and potential energy) can cause the attract force between them. The constant $\mathrm{G}_{\mathrm{c}}\left(8.26269 \times 10^{-45} \mathrm{~m}^{-1} \mathrm{~kg}^{-1} \mathrm{~s}^{2}\right)$ was given in the equation. The equation may help to explain and understand the phenomena of basis physics, cosmology and astronomy, such as the dark matter or dark energy.
\end{abstract}

Keywords: Attract force, Energy, Equation

\section{Introduction}

The dark matter or dark energy problem were cared in cosmology and astronomy, which concerted cosmos original and globe actions ${ }^{[1,2]}$. Until now, the dark energy and dark matter were a hypothesis. Since the 1990s, the observations data indicating that the universe is accelerated expanding. Based on the standard model of cosmology, on a mass-energy equivalence $\left(\mathrm{E}=\mathrm{mc}^{2}\right)$ basis, on Planck mission team, and on the observable data, that the universe contains $68.3 \%$ dark energy, $26.8 \%$ dark matter, and $4.9 \%$ ordinary matter. So it is important to find and determine the dark energy and dark matter. ${ }^{[3,4,5]}$

Newton's law of universal gravitation (Eq.1) states that any two bodies in the universe attract each other with a force that is directly proportional to the product of their masses and inversely proportional to the square of the distance between them. Separately it was shown that large spherically symmetrical masses attract and are attracted as if all their mass were concentrated at their centers. This is a general physical law derived from empirical observations by what Isaac Newton called induction. It is a part of classical mechanics and was formulated in Newton's work "Philosophiæ Naturalis Principia Mathematica", first published on 5 July $1687^{[6,7]}$

Einstein mass-energy equivalence (Eq.2) ${ }^{[8,9,10]}$ is the concept that the mass of an object or system is a measure of its energy content. In physical system have two basis properties: one is energy, and the other is mass. These two properties are always both present constant relationship or in the same proportion to one another. Albert Einstein developed the mass-energy equivalence that energy $\mathrm{E}$ and mass $\mathrm{m}$ is reliant on the speed of light $\mathrm{c}$, which was in 1905 in one of his "Annus Mirabilis" papers entitled "Does the inertia of an object depend upon its energy content?" ${ }^{[8]}$.

The re-considering of Newton force principle and Einstein matter energy equation may get some new knowledge, which may help to understand the dark matter or dark energy phenomena, or other related energy forms. This paper deduces an equation for energy attraction and tries to give a new concept to view the matter, energy, and force. These equations may help to explain and understand the phenomena of basis physics, cosmology and astronomy, such as dark energy, dark matter, and black holes.

\section{Results and Discussions}

The Newton the principle of gravitation (Eq.(1)) open out the gravitation force between two matter ${ }^{[11]}$. This means that matter produce attract force. The equation (Eq.(2))of Einstein open out the relationship between matter and energy ${ }^{[12,13]}$. This mean matter has energy or energy properties. Then if consider the Eq.(1) with Eq. (2), and connect the two equation, there may got a new equation relationship. Consider further, if there are attract force between two energy fields, which means 
a new view on matter, energy, and force. So the Eq.(7) and Eq.(8) were got, which found the relationship between attract force and energy. And the equation may explain the phenomena of the dark matter or dark matter in cosmology and astronomy ${ }^{[14]}$. Eq.(7) and Eq. (8) have general meaning to consider the force with energy.

Newton the principle of gravitation was in Eq.(1):

$$
F=G \frac{M_{1} M_{2}}{r^{2}}
$$

Einstein matter energy equation was in Eq.(2):

$$
E=M c^{2}
$$

The equation (Eq.(2)) of Einstein mass-energy can be written in Eq.(3).

$$
M=E / c^{2}
$$

The Eq.(4) is got by putting the Eq.(3) into Eq.(1).

$$
F=G \frac{\left(E_{1} / c^{2}\right)\left(E_{2} / c^{2}\right)}{r^{2}}
$$

Changing the form of Eq.(4) into Eq.(5).

$$
F=\frac{G}{c^{2} c^{2}} \frac{E_{1} E_{2}}{r^{2}}
$$

The constant $\mathrm{G}_{\mathrm{c}}$ is ordered by Eq.(6).

$$
G_{c}=\frac{G}{c^{2} c^{2}}=\frac{G}{c^{4}}
$$

Then the Eq.(7) is got.

$$
F=G_{c} \frac{E_{1} E_{2}}{r^{2}}
$$

If only replace one M in Eq.(1) by Eq.(3), the Eq.(8) can be got.

$$
F=G \frac{M_{1}\left(E_{2} / c^{2}\right)}{r^{2}}
$$

The $\mathrm{F}$ is the force. The $\mathrm{M}$ is the mass of matter. The $\mathrm{c}$ is the speed of light. The $r$ is the distance between the two matters or two energy fields. The $\mathrm{E}$ is energy. The $\mathrm{G}$ is the gravitation constant. The $\mathrm{G}_{\mathrm{c}}$ is the constant of gravitation of energy.

The $G_{c}$ in Eq.(7) is a constant get from Eq.(6). The gravitation constant $(\mathrm{G})^{[15]}$ is about $(6.67428 \pm 0.00067) \times 10^{-11}$ $\mathrm{m}^{3} \mathrm{~kg}^{-1} \mathrm{~s}^{-2}$. The $\mathrm{G}_{\mathrm{c}}$ is got from $\mathrm{G}$ divided by the biquadrate of light's speed $(c=299,792,458 \mathrm{~m} / \mathrm{s})$. So the constant $G_{c}$ is quite small $\left(8.26269 \times 10^{-45} \mathrm{~m}^{-1} \mathrm{~kg}^{-1} \mathrm{~s}^{2}\right)$. Consider the distance $(\mathrm{r})$ of the two energy fields and constant $G_{c}$ in Eq.(7), the attract force $(F)$ caused by energy exists but small.

The $r$ is distance between two energy bodies. Energy has no spatial dimensions, but energy has its field ranges. Such as photon, electronic fields, magnetic fields, and some other energy forms, that have energy field ranges. The $r$ means the distance of two energy field centers.

The Eq.(8) can show the attract force between the matter and energy fields. So from Eq.(7) and Eq.(8) it can be concluded that there is attract force between the two energy fields, or between the matter and energy fields. Eq.(1) means there force between matter, Eq.(2) mean matter has energy, so using the energy to replace the matter in Eq.(1), then giving out Eq. (7) and Eq.(8). Energy has lots of forms, such as light, photon, heat, fields, and kinetic energy or potential energy. The different forms energy always acts on each other and on matter, which through force or fields to carry out these actions. So it was not difficult to think that there exist force between two energy bodies $\left(E_{1}\right.$ and $\left.E_{2}\right)$, or between one energy body $\left(E_{1}\right)$ and one matter body $\left(M_{1}\right)$.

There are many energy forms in cosmic, such as black hole, galaxy, nebula, and undetermined dark energy. The black hole always exists at the center of galaxy, and have large attract force. It was deduced by researchers that black hole has large density, large matter, or large energy. By using Eq. (8), large energy black hole can attract stars, planet, dust, or other matter body. The black hole as its named black was that can attract light to be difficult detected by optical telescope, only except $\boldsymbol{\gamma}$ - or X- rays. The Eq.(7) can explain that large energy black holes attract light form energy to prevent the light flee from black hole fields ranges.
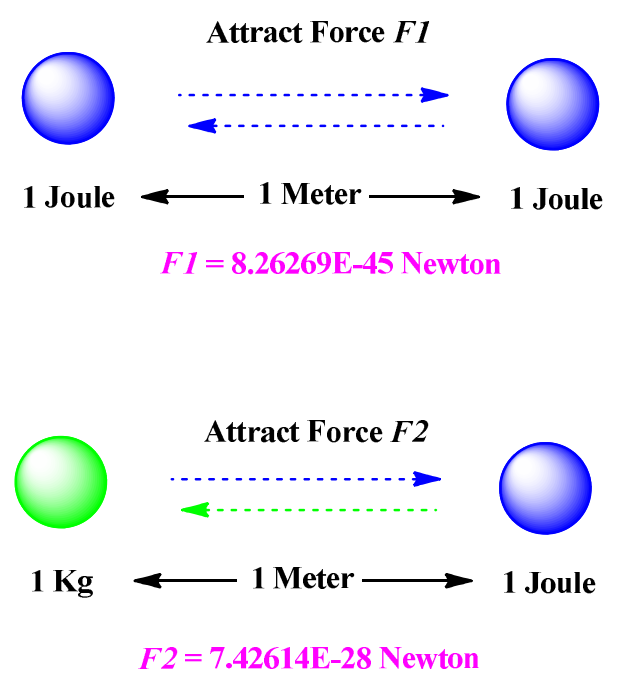

Figure 1. The illumination example of attract force (above: two energy bodies) (below: one matter body with one energy body).

For example in Figure 1, the attract force exists between two energy bodies or between one matter body and one energy body. One Joule energy body has $8.26269 \times 10^{-45}$ Newton attraction force with the other one Joule energy body from one Meter away (calculated from Eq. (7)). One Kilogram matter body has $7.42614 \times 10^{-28}$ Newton attraction force with the other one Joule energy body from one Meter away (calculated from Eq. (8)). The attract force got in Figure 1 was small, for the E-45 or E-28 magnitude. The 
example was 1 Joule and 1Kilogram. The celestial body matter or energy field in cosmology and astronomy was very large to produce high attract force, which take act on matter or energy filed body in cosmos.

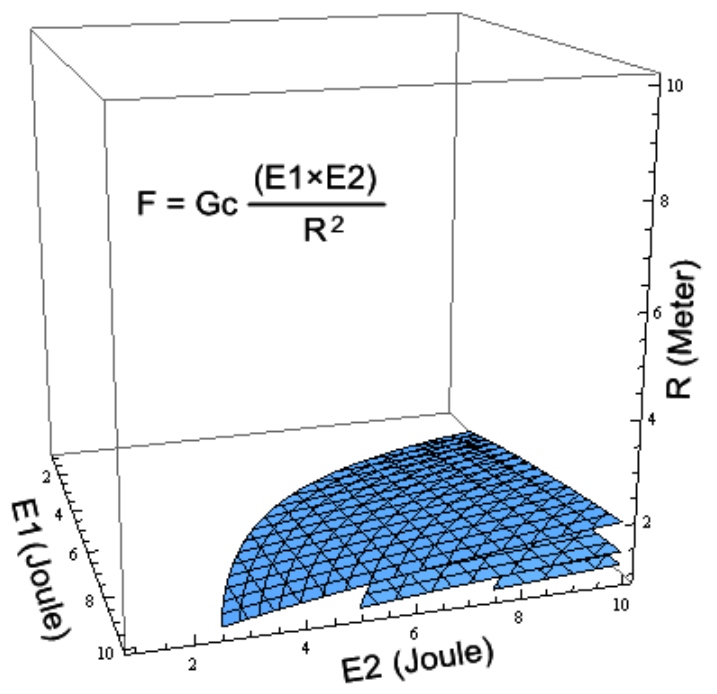

Figure 2. The function related three-dimension contour of Eq.(7) (blue contour was $F \times 10^{45}$ ).

The Figure 2 show the functional relationship of Eq.(7). This three dimensions contour was $\mathrm{F} \times 10^{45}$, which reflect the attract force (F) situations between two energy bodies (E1, E2) in $r$ distance.

The Eq.(7) may help to explain the phenomenon of the dark matter in cosmos. The attract force can be caused by the energy in cosmos. There are lots of heat energy and light energy which take part in the being of the attract force in cosmos. There are some other conclusions may be got that electronic energy, magnetic energy, kinetic energy or potential energy could also cause the attract force in some forms.

\section{Conclusion}

Deduced from Newton the principle of gravitation and Einstein matter energy equation, the Eq.(7) can explain that there is attract force between the two energy fields. The attract force is not only cause by matter but also by energy. The energy may be any forms, such as the energy of heat, the energy of photon, the electronic energy, the magnetic energy, even the kinetic energy, and the potential energy. The Eq.(7) and Eq.(8) release that there is attract force between two energy fields or between matter and energy fields. The equations may help to explain the dark matter or dark energy phenomenon.

\section{References}

[1] F. Zwicky, "Die Rotverschiebung von extragalaktischen Nebeln." Helvetica Physica Acta. 1933, 6,110-127..

[2] F. Zwicky, "On the Masses of Nebulae and of Clusters of Nebulae.” The Astrophysical Journal. 1937, 86, 217.

[3] Ade, P. A. R.; Aghanim, N.; Armitage-Caplan, C.; et al. (Planck Collaboration) (22 March 2013). "Planck 2013 results. I. Overview of products and scientific results - Table 9.". Astronomy and Astrophysics

[4] Ade, P. A. R.; Aghanim, N.; Armitage-Caplan, C.; et al. (Planck Collaboration) (31 March 2013). "Planck 2013 Results Papers". Astronomy and Astrophysics

[5] "First Planck results: the Universe is still weird and interesting". Sean Carroll, Ph.D., Cal Tech, 2007, The Teaching Company, Dark Matter, Dark Energy: The Dark Side of the Universe, Guidebook Part 2 page 46, Accessed Oct. 7, 2013,

[6] Walter Lewin (October 4, 1999). Work, Energy, and Universal GravitatioT Course 8.01: Classical Mechanics, Lecture 11. (ogg) (videotape) (in English). Cambridge, MA USA: MIT OCW.

[7] Isaac Newton: "In [experimental] philosophy particular propositions are inferred from the phenomena and afterwards rendered general by induction": "Principia", Book 3, General Scholium, at p.392 in Volume 2 of Andrew Motte's English translation published 1729 .

[8] Einstein, A. Ist die Trägheit eines Körpers von seinem Energieinhalt abhängig?, Annalen der Physik, 1905,18 (13): 639-643,

[9] Lev B.Okun, The concept of Mass, Physics Today 42 (6), June 1969, p. 31-36,

[10] Paul Allen Tipler, Ralph A. Llewellyn (January 2003), Modern Physics, W. H. Freeman and Company, pp. 87-88,

[11] I. Bernard Cohen and Anne Whitman, “translators:Isaac Newton , The Principia: Mathematical Principles of Natural Philosophy. Preceded by A Guide to Newton's Principia” University of California Press 1999. Proposition 75, Theorem 35: p.956

[12] R. Tolman, Philosophical Magazine, 1912, 23, 375.

[13] E. F. Taylor, J. A. Wheeler, "Spacetime Physics" second edition. New York: W.H. Freeman and Company. 1992.

[14] D. Clowe, M. Bradac, A. H. Gonzalez et al. “A direct empirical proof of the existence of dark matter.” Astrophys J Lett. 2006, 648,109 .

[15] George T. Gillies, “The Newtonian gravitational constant: recent measurements and related studies" Progress in Physics., $1997,60,151$ 